\title{
A UNIFIED APPROACH TO UNIQUENESS OF WALSH SERIES AND HAAR SERIES
}

\author{
WILLIAM R. WADE
}

\begin{abstract}
We obtain a uniqueness theorem for Walsh series valid for subsequences of $2^{n}$ th partial sums which satisfy a pointwise growth condition.
\end{abstract}

1. Introduction. There are three techniques for obtaining uniqueness results for Walsh series: quasimeasures, formal integration, and nested intervals.

The quasimeasure technique, introduced by Yoneda (see [8]), is designed to obtain uniqueness results for Walsh series which are everywhere finite but satisfy no other growth condition.

The formal integral technique, originated by Riemann for trigonometric series, adapted for Walsh series by Fine [4], and developed by many others (e.g., Crittenden and Shapiro [3], and Lindahl [5]) obtains uniqueness results for Walsh series satisfying pointwise growth conditions, but does not apply to subsequences of $2^{n}$ th partial sums.

The nested interval technique, introduced by Arutunjan and Talaljan [2], and developed by others (e.g., Skvorcov [6]), applies to subsequences of $2^{n}$ th partial sums, but obtained results only for Walsh series satisfying uniform growth conditions. This situation prevailed because the technique primarily involved Haar series, with the Walsh series results obtained as corollaries (e.g., see [7]).

Here, we modify this technique so that it handles pointwise growth conditions and applies directly to Walsh series. The resulting uniqueness theorem is powerful and general, surpassing many earlier results of all three types.

2. Statement of results. Let $w_{0}, w_{1}, \ldots$ denote the Walsh functions and let $G$ denote the dyadic group (see [4]). For each $f$ integrable on the group $G$, let $S[f]$ denote the Walsh-Fourier series of $f$. For each Walsh series $S=\sum a_{k} w_{k}$, let

$$
S_{2^{n}}=\sum_{k=0}^{2^{n}-1} a_{k} w_{k}, \quad n=0,1, \ldots
$$

denote the $2^{n}$ th partial sums of $S$.

A Walsh series is said to satisfy the C-S (Crittenden-Shapiro) condition if

$$
\lim _{n \rightarrow \infty} 2^{-n} S_{2^{n}}(x)=0
$$

for all $x \in G$. We shall call a Walsh series singular if there is an $x \in G$ such that

$$
2^{-n} S_{2^{n}}(x) \equiv d
$$

Received by the editors December 3, 1985.

1980 Mathematics Subject Classification (1985 Revision). Primary 42C10; Secondary 43A75.

Key words and phrases. Walsh series, Haar series, uniqueness.

This research was supported in part by a National Science Foundation grant (NSF-INT8400708). 
for some nonzero constant $d$, and all $n$ sufficiently large. Thus every Walsh series which satisfies the C-S condition is nonsingular.

In the next section we shall prove the following result.

THEOREM 1. Let $f$ be integrable on $G, n_{1}, n_{2}, \ldots$ be a subsequence of nonnegative integers, and $S$ be a Walsh series which satisfies

$$
\limsup _{j \rightarrow \infty}\left|S_{2_{j}^{n}}(x)\right|<\infty
$$

for all but countably many $x \in G$. If $S-S[f]$ is nonsingular, and

$$
\lim _{j \rightarrow \infty} S_{2_{j}^{n}}=f
$$

a.e. on $G$, then $S$ is the Walsh-Fourier series of $f$.

Let $x \in G$ and recall that for each integer $n>0$ there are sets $I_{n}$ of Haar measure $2^{-n}$ such that

$$
2^{-n} S_{2^{n}}[f, x]=\int_{I_{n}} f(t) d t
$$

Hence every Walsh-Fourier series satisfies the C-S condition, and Theorem 1 is true if " $S-S[f]$ is nonsingular" is replaced by " $S$ satisfies the C-S condition." In particular, the following result is true:

Corollary 1 (Theorem 1 OF [3] AND TheORem 4 OF [5]). If $S$ is a Walsh series which satisfies the $C$-S condition whose $2^{n}$ th partial sums converge as $n \rightarrow \infty$, except possibly on a countable subset of $G$, to a finite-valued, integrable function $f$, then $S$ is the Walsh-Fourier series of $f$.

Instead of $w_{0}, w_{1}, \ldots$ and the group $G$, some authors have studied Walsh functions $\psi_{0}, \psi_{1}, \ldots$ defined on the interval $[0,1]$. Fine's map $\lambda: G \rightarrow[0,1]$ can be used to translate results from the $w_{k}$ 's to the $\psi_{k}$ 's. Indeed (see [4]), there is a countable subset $Z$ of $G$ such that $\lambda$ is 1-1 from $G \sim Z$ onto [0,1), 1-1 from $Z$ onto the dyadic rationals in $[0,1]$, and such that

$$
\begin{aligned}
& w_{k}(x)=\psi_{k}(\lambda(x)), \quad x \in G \sim Z, \\
& w_{k}(x)=\psi_{k}(\lambda(x)-0), \quad x \in Z,
\end{aligned}
$$

both hold for $k=0,1, \ldots$.

Given any series $S$ defined on $[0,1]$, we shall call $S$ singular (on $[0,1]$ ) if there is an $x \in[0,1]$ such that $2^{-n} S_{2^{n}}(x \pm 0)=d$ for some nonzero constant $d$, some choice of \pm , and all $n$ sufficiently large. Similarly, we say that $S$ satisfies the C-S condition (on $[0,1])$ if

$$
\lim _{n \rightarrow \infty} 2^{-n} S_{2^{n}}(x \pm 0)=0
$$

for all $x \in[0,1]$. Since each $\psi_{k}$ is continuous on the dyadic irrationals, and continuous from the right on $[0,1)$, it is clear that a Walsh series $\sum a_{k} \psi_{k}$ is singular (respectively, satisfies the C-S condition) on $[0,1]$ if and only if the Walsh series $\sum a_{k} w_{k}$ is singular (respectively, satisfies the C-S condition) on the group $G$. In particular, Theorem 1 and Corollary 1 are also true for Walsh series defined on $[0,1]$.

Since each Walsh function takes on only the values \pm 1 , Schwartz' inequality implies that any Walsh series whose coefficients tend to zero necessarily satisfies the C-S condition. Therefore, Theorem 1 also contains the following result: 
COROLlARY 2 (THEOREM 2 OF [2]). Let $S$ be a Walsh series whose coefficients $a_{k}$ tend to zero as $k \rightarrow \infty$, and let $f$ be a finite-valued, integrable function on $[0,1]$. If some subsequence $S_{2_{j}^{n}}(x)$ converges to $f(x)$ as $j \rightarrow \infty$, for all but countably many $x \in[0,1]$, then $S$ is the Walsh-Fourier series of $f$.

Applying Theorem 1 to $f=0$, the following new result eventuates:

COROLlARY 3. If $S$ is a nonsingular Walsh series, $n_{1}, n_{2}, \ldots$ is a subsequence of nonnegative integers such that $S_{2_{j}^{n}} \rightarrow 0$ a.e. as $j \rightarrow \infty$, and (3) holds for all but countably many $x \in[0,1]$, then $S$ is the zero series. let

Let $h_{0}, h_{1}, \ldots$ denote the Haar functions and, for each Haar series $H=\sum c_{k} h_{k}$,

$$
H_{n} \equiv \sum_{k=0}^{n-1} c_{k} h_{k}
$$

denote the $n$th partial sums. Recall (see [1]) that if $2^{m} \leq n<2^{m+1}$ and $n=2^{m}+k$ then

$$
H_{n}(x)= \begin{cases}H_{2^{m}}(x), & k / 2^{m}<x<1, \\ H_{2^{m+1}}(x), & 0<x<k / 2^{m} .\end{cases}
$$

Also recall that there is a 1-1 correspondence from Haar series $H$ to Walsh series $S$ defined on $[0,1]$ which takes Haar-Fourier series to Walsh-Fourier series such that

$$
H_{2^{m}}(x) \equiv S_{2^{m}}(x), \quad m=0,1, \ldots,
$$

for all dyadic irrational $x \in(0,1)$. Since the collection of dyadic rationals is countable, the following new result is an immediate consequence of Theorem 1.

COROLlaRY 4. Let $H$ be a Haar series which satisfies the $C-S$ condition on $[0,1]$, let $n_{1}, n_{2}, \ldots$ be a subsequence of integers and suppose

$$
\limsup _{j \rightarrow \infty}\left|H_{n_{j}}(x)\right|<\infty
$$

for all but countably $x \in[0,1]$. If $H_{n_{j}}$ converges a.e. to $f$ on $[0,1]$ as $j \rightarrow \infty$, then $H$ is the Haar-Fourier series of $f$.

A Haar series $\sum c_{k} h_{k}$ is said to satisfy the A-T (Arutunjan-Talaljan) condition if for each $x \in[0,1]$,

$$
\lim _{l \rightarrow \infty} \frac{c_{k_{l}}}{h_{k_{l}}(x)}=0
$$

where $k_{1}, k_{2}, \ldots$ represent those indices $k$ for which $h_{k}(x) \neq 0$. Since each $h_{k}=$ $O\left(\sqrt{2^{n}}\right)$ as $k \rightarrow \infty$, where $2^{n} \leq k<2^{n+1}$, it is easy to see that every Haar series $S$ which satisfies the A-T condition satisfies the C-S condition. Hence Corollary 4 contains the following one:

COROLlaRY 5 (THEOREM 1 OF $[2]$ ). Let $f$ be a finite-valued integrable function on $[0,1]$, let $n_{1}, n_{2}, \ldots$ be a subsequence of integers, and let $H$ be a Haar series satisfying the $A-T$ condition. If $H_{n_{j}}(x)$ converges to $f(x)$ as $j \rightarrow \infty$, for all but countably many $x \in[0,1]$, then $H$ is the Haar-Fourier series of $f$.

It should be noted that following Skvorcov [6], the techniques of the next section can be modified to handle the case when $f$ is Denjoy integrable in the narrow sense. In particular, Theorem 1 and its five corollaries remain valid if "Denjoy integrable in the narrow sense" replaces "integrable." 
3. A proof of Theorem 1. For simplicity, let $n_{j}=j$. Let $S[f]$ represent the Walsh-Fourier series of $f$ and set $T=S-S[f]$.

Let $G=G_{0} \supset G_{1} \supset \cdots$ represent the canonical neighborhood base at 0 in $G$. Thus each $G_{m}$ is a closed subgroup of Haar measure $2^{-m}$. For each integer $m \geq 0$ we shall denote the collection of distinct cosets of $G_{m}$ by $I(p, m), p=0,1, \ldots, 2^{m}-1$. We shall say that $I$ and $J$ represent different halves of $I(p, m)$ if $I$ and $J$ are cosets of $G_{m+1}, I \cap J=\varnothing$, and $I \cup J=I(p, m)$.

Suppose the theorem is false. Then there exist integers $p_{0}, m_{0}$ such that $T_{2^{m_{0}}} \neq 0$ on $I_{0} \equiv I\left(p_{0}, m_{0}\right)$. Since Walsh functions of order less than $2^{m_{0}}$ are constant on $I_{0}$ we have

$$
T_{2^{m_{0}}} \equiv d \neq 0 \quad \text { on } I_{0} .
$$

Let $I_{1}, J_{1}, I_{2}, J_{2}, \ldots$ be any sequence of cosets such that, for each $k>0, I_{k}$ and $J_{k}$ represent two different halves of $I_{k-1}$. We claim that there is an integer $k$ such that if $I\left(p_{1}, m_{1}\right)=J_{k}$ then

$$
T_{2^{m_{1}}} \neq 0 \text { on } I\left(p_{1}, m_{1}\right) .
$$

To prove this claim, suppose to the contrary that $T_{2^{m_{0}+k}} \equiv 0$ on $J_{k}$ for $k=$ $1,2, \ldots$ For $k=1$ we have by (5) that

$$
T_{2^{m_{0}}}-T_{2^{m_{0}+1}}=d \text { on } J_{1} \text {. }
$$

Since for each $2^{m_{0}} \leq l<2^{m_{0}+1}$ the Walsh function $w_{l}$ changes signs from $J_{1}$ to $I_{1}$, it follows from (7) and (5) that $T_{2^{m_{0}+1}} \equiv 2 d$ on $I_{1}$. By induction,

$$
T_{2^{m_{0}+k}} \equiv 2^{k} d \quad \text { on } I_{k}, k \geq 0 .
$$

By assumption, the cosets $I_{k}$ form a nested sequence of compact sets in $G$. Hence there exists a point $\xi$ which belongs to $I_{k}$ for all $k \geq 0$. Consequently (8) implies $2^{-k} T_{2^{m_{0}+k}}(\xi) \equiv d$ for all $k \geq 0$. It follows that $T$ is singular, contrary to hypothesis. Therefore the claim is established.

Let $x_{1}, x_{2}, \ldots$ represent the set of points at which (3) fails to hold. If $x_{1}$ does not belong to $I_{0}$, let $I_{1}, J_{1}, I_{2}, J_{2}, \ldots$ be any sequence of cosets such that, for each $k>0, I_{k}$ and $J_{k}$ represent different halves of $I_{k-1}$. If $x_{1}$ belongs to $I_{0}$, choose the cosets $I_{1}, J_{1}, I_{2}, J_{2}, \ldots$ with the additional property that $x_{1} \in I_{k}$ for all $k \geq 0$. Hence $x_{1} \notin J_{k}$ for all $k \geq 1$ and it follows from the claim that there exist integers $p_{1}$ and $m_{1}$ such that $I\left(p_{1}, m_{1}\right)$ is contained in $I\left(p_{0}, m_{0}\right)$ but does not contain $x_{1}$ and (6) holds. Moreover, since $S_{2^{m}}[f] \rightarrow f$ in $L^{1}$ norm, as $m \rightarrow \infty$, it is clear that $\left|S_{2^{m}}\right| \leq 1+\left|S_{2^{m}}[f]\right|$ on $I\left(p_{1}, m_{1}\right)$ for $m \geq m_{1}$ together with (6), the orthogonality of the Walsh system, and the bounded convergence theorem imply

$$
\begin{aligned}
0 & \neq \int_{I\left(p_{1}, m_{1}\right)} T_{2^{m_{1}}}(x) d x \\
& =\lim _{m \rightarrow \infty} \int_{I\left(p_{1}, m_{1}\right)} T_{2^{m}}(x) d x=0 .
\end{aligned}
$$

Therefore, we may suppose $\left|S_{2^{m_{1}}}\right|>1+\left|S_{2^{m_{1}}}[f]\right|$ on $I\left(p_{1}, m_{1}\right)$. Since $\left|T_{2^{m_{1}}}\right| \geq$ $\left|S_{2^{m_{1}}}\right|-\left|S_{2^{m_{1}}}[f]\right|$ we still have $T_{2^{m_{1}}} \neq 0$ on $I\left(p_{1}, m_{1}\right)$, and can apply the claim again. 
Continuing in this manner, we generate a nested sequence of cosets $I\left(p_{k}, m_{k}\right)$ such that

$$
x_{k} \notin I\left(p_{k}, m_{k}\right)
$$

and $\left|S_{2^{m_{k}}}\right|>k+\left|S_{2^{m_{k}}}[f]\right| \geq k$ on $I\left(p_{k}, m_{k}\right)$, for $k=1,2, \ldots$ Consequently, if $x_{0} \in I\left(p_{k}, m_{k}\right)$ for all $k \geq 1$, then

$$
\limsup _{m \rightarrow \infty}\left|S_{2^{m}}\left(x_{0}\right)\right|=\infty,
$$

i.e., $x_{0}=x_{k}$ for some $k$. Since this contradicts (9), we conclude that $S$ is the Walsh-Fourier series of $f$.

We close by noting that nonsingularity of $S$ was used only to avoid the countable set where (3) fails to hold. In particular, our proof also establishes the following result:

THEOREM 2 (THEOREM 1 OF [8]). Let $S$ be a Walsh series which satisfies

$$
\limsup _{j \rightarrow \infty}\left|S_{2^{n_{j}}}(x)\right|<\infty
$$

for all $x \in G$. If $S_{2^{n_{j}}}$ converges a.e. to an integrable function $f$ on $G$, then $S$ is the Walsh-Fourier series of $f$.

\section{REFERENCES}

1. G. Alexits, Convergence problems of orthogonal functions, transl. by I. Földes, Pergamon Press, New York, 1961.

2. F. G. Arutunjan and A. A. Talajan, On uniqueness of Haar and Walsh series, Izv. Akad. Nauk SSSR 28 (1964), 1391-1408.

3. R. B. Crittenden and V. L. Shapiro, Sets of uniqueness on the group $2^{\omega}$, Ann. of Math. 81 (1965), 550-564.

4. N. J. Fine, On the Walsh functions, Trans. Amer. Math. Soc. 65 (1949), 372-414.

5. R. Lindahl, A differentiation theorem for functions defined on dyadic rationals, Proc. Amer. Math. Soc. 30 (1971), 349-352.

6. V. A. Skvorcov, Haar series with convergent subsequences of partial sums, Dokl. Akad. Nauk SSSR 183 (1968), 784-786.

7. W. R. Wade, $A$ uniqueness theorem for Haar and Walsh series, Trans. Amer. Math. Soc. 141 (1969), 187-194.

8. W. R. Wade and K. Yoneda, Uniqueness and quasi-measures on the group of integers of a p-series field, Proc. Amer. Math. Soc. 84 (1982), 202-206.

Department of Mathematics, University of Tennessee, Knoxville, TenNESSEE 37996-1300 